Review Article

\title{
Expert consensus on defining sensitive skin and role of cosmeceuticals: an Indian perspective
}

\author{
Malavika Kohli ${ }^{1 *}$, Hema Pant ${ }^{2}$, Rasya Dixit ${ }^{3}$, Anchala Parthasaradhi ${ }^{4}$ \\ ${ }^{1}$ Department of Dermatology, Dermatology and Aesthetics Mumbai, Maharashtra, India \\ ${ }^{2}$ SCULPT Aesthetic and Cosmetic clinic, Defence Colony, New Delhi, India \\ ${ }^{3}$ Cosmetic Dermatology Bangalore, Karnataka, India \\ ${ }^{4}$ Skin Institute \& Research Center, Hyderabad, Telangana, India
}

Received: 10 September 2020

Accepted: 06 October 2020

*Correspondence:

Dr. Malavika Kohli,

E-mail: malavikakohli@gmail.com

Copyright: (C) the author(s), publisher and licensee Medip Academy. This is an open-access article distributed under the terms of the Creative Commons Attribution Non-Commercial License, which permits unrestricted non-commercial use, distribution, and reproduction in any medium, provided the original work is properly cited.

\begin{abstract}
In clinical practice, the 'sensitive skin' phenomenon, encompassing a wide range of signs and symptoms, is majorly a self-diagnosed condition. Various articles have given perspective on the heterogeneous concept of sensitive skin in varied ways. Cosmeceuticals, commonly used for sensitive skin, contain biologically active ingredients that deliver multifunctional skin benefits. Since no guidelines have been formulated for the use of cosmeceutical products in the management of sensitive skin, an individualized skin care regimen is usually adopted. Also, cosmeceutical products have been demonstrated to play a crucial role as add-on therapy in the management of dermatological disorders. However, there exists uncertainty about sensitive skin manifestation and well-defined use of cosmeceuticals for sensitive skin. This paper provides a consensus clinical viewpoint of expert clinicians, which will help clear the pathway for effective identification and management of sensitive skin using cosmeceuticals.
\end{abstract}

Keywords: Sensitive skin, Cosmeceuticals, Cleanser, Moisturizer, Sunscreen, Shampoo

\section{INTRODUCTION}

Sensitive skin is a general term used to describe skin discomfort and/or irritation. It is frequently referred to as hypersensitivity, hyperreactivity, intolerance or irritation of the skin. In 1987, Maibach first described 'sensitive skin' under the term 'Cosmetic Intolerance Syndrome'. Epidemiological studies and patient reports have ascertained that sensitive skin, apart from being a cosmetic intolerant concern, may result from several intrinsic and extrinsic factors. ${ }^{1}$ Thus, a gamut of causative factors is currently known to contribute to the manifestation of sensitive skin syndrome. An individual may complain of symptoms such as burning, stinging, itching, and dryness, and these symptoms can be perceived by them as skin sensitivity. ${ }^{2}$ More importantly, these self-perceived symptoms may or may not be warranted by the presence of any objective signs such as visible clinical lesions on the skin. In the real-world scenario, sensitive skin is, in fact, a self-diagnosed condition usually lacking objective clinical findings. ${ }^{1,2}$

Given the heterogeneity of appearance and perception, it is difficult to define or classify the term 'sensitive skin'. There have also been numerous attempts to define 'sensitive skin' in order to summarize the scope of clinical and causal diversity. ${ }^{1,2}$ The purpose of this consensus document is to provide dermatologists' expert opinion on sensitive skin and role of cosmeceuticals in it.

\section{Need for consensus}

Heterogenous manifestation and perception of sensitive skin, existence of multifactorial clinical and causal 
ambiguity, defining sensitive skin is challenging and no definite indication for cosmeceutical use in people with sensitive skin

\section{METHODS}

The experts' group meeting, including 40 dermatologists, was held in four major cities of India - Delhi, Mumbai, Bangalore, and Hyderabad. The main purpose of the meeting was to understand the concept of sensitive skin in Indians of sensitive skin and role of cosmeceuticals in its management. The two main objectives of this focused group meeting were:

To review the literature thoroughly and systematically, covering various aspects of sensitive skin concept such as definition, epidemiology, causative factors, clinical features, diagnostic tests, and management using cosmeceutical products for daily skin care regimen.

To discuss the evidence-based global and Indian concept of sensitive skin and use of cosmeceuticals in its management.

Thereafter, clinical insights were drawn from the dialogue with an attempt to derive clinically relevant experts' opinion and achieve a consensus standpoint.

We performed a literature searches using PubMed, a Google and Google scholar. We identified relevant articles using Boolean operators AND/OR for search terms/keywords like sensitive skin, cosmeceuticals, prevalence, pathophysiology, treatment, management, cleansers, moisturizers, sunscreen, shampoo, and skin care. Using this strategy, 41 published literature in English in the form of research articles, systematic reviews or meta-analyses, corresponding to human subjects were used for the preparation of this consensus paper.

This paper summarizes experts' discussion on evidencebased literature, and provides a consensus clinical view at defining, diagnosing, and managing sensitive skin with appropriate cosmeceuticals. The consensus draft has been formulated in accordance with the experts' observations and suggestions. Each section in the article is followed by consensus points for better understanding the experts' perspective.

\section{DEFINING SENSITIVE SKIN}

Based on the literature review, sensitive skin is defined as the subjective identification of skin discomfort in the absence of visible skin lesions. ${ }^{3}$ However, some people may have adverse reactions to the use of certain products or irritants due to an exaggerated or higher sensitivity of their skin. ${ }^{3}$ Sensitive skin refers to a sensory reaction triggered by contactors and/or environmental factors, usually, without a visible clinical manifestation. ${ }^{1}$ Sensitive skin generally manifests in two forms- objective and subjective (Figure 1). In the objective form, clinical lesions are visible such as erythema, papules, redness, and scaling. On the other hand, in the subjective form, a patient only describes the symptoms in the absence of clinically visible signs.1Thus, it is important to define the term 'sensitive skin'.
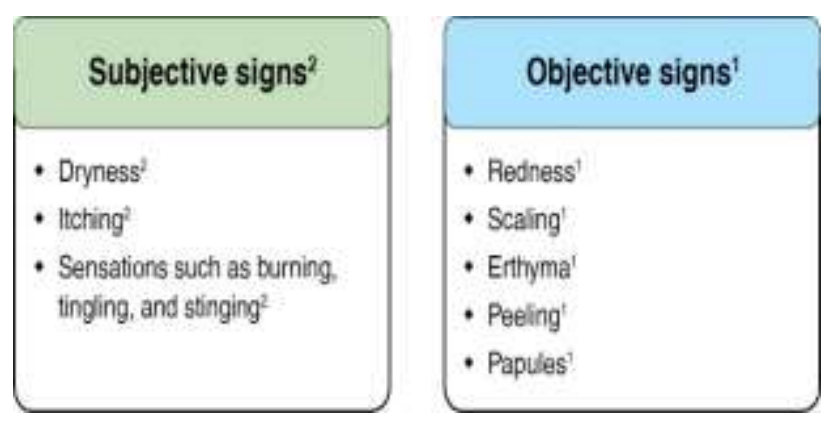

Figure 1: Subjective and objective signs of sensitive skin.

Nevertheless, in an individual with sensitive skin, one or multiple parameters could be present. The following are different descriptions of sensitive skin to characterize its phenomenon. 1) Subjective irritation - irritant response without visible clinical signs. ${ }^{4}$ 2) Neurosensory irritationnaturally mediated responses such as itching, stinging, burning, tightness. ${ }^{4}$ 3) Chemosensory - sensory responses induced by chemicals in contrast to physical, mechanical, and environmental factors. ${ }^{4}$ 4) Psychophysical irritation psychological component is involved. ${ }^{4}$

\section{CONSENSUS KEY POINT 1}

The panel agreed that sensitive skin could be accurately defined only when both subjective and objective perception are evaluated. Patients first provide subjective description of the symptoms following the use of specific skin care products or other triggering factors. Thereafter, a clinician can examine the symptoms to identify and evaluate any underlying skin pathology pertaining to the symptoms. Thus, a complete and clinically acceptable definition of sensitive skin should consider both subjective perception and objective assessment of the skin and symptoms.

\section{Epidemiology}

Epidemiological studies report prevalence of sensitive skin to be $50 \%$ to $61 \%$ in women and $30 \%$ to $44 \%$ in men. ${ }^{1}$ There is clinical evidence supporting the fact that sensitive skin is more prevalent in young people. In older people, integrity of skin tissue changes and tactile sensation decreases, thus, diminished response to sensitivity is observed. ${ }^{1}$

Studieson Indian population reportsensitive skin to be a frequent condition in women and men, affecting about 1/3rd of the population..$^{5}$ An Indian epidemiological study (Brenaut $\mathrm{E}$ et al) in a representative nationwide sample 
$(n=3012$, age $\geq 15)$ evaluated the prevalence of sensitive skin in Indian male and female population. The study reported that sensitive skin was frequent in younger subjects (15-44 years old, $32.4 \%)$ than in older subjects (over 45 years old, $24.2 \%, \mathrm{p}<0.001) .^{5}$

\section{CONSENSUS KEY POINT 2}

According to the experts' clinical practice, sensitive skin is observed to be more common in the 16-60 yeas age group. Though it is difficult to have a concrete evidence base on the discussion, the experts agreed that a greater number of females are reported to have sensitive skin than males. The factors that may explain the high prevalence of sensitive skin in women are- hormonal changes, thin skin, excessive cosmetic use, and more awareness regarding products and sensitive skin issues. In men, sensitive skin is under-reported. Besides triggers mentioned above, in many cases, other factors such as occupation and overexposure to pollutants are also observed.

\section{Etiology}

Sensitive skin is a multifactorial syndrome. In many people, hyperreactivity is genetic but there are many factors, endogenous or exogenous, that can trigger or aggravate the condition. ${ }^{1}$ (Figure 2) shows various predisposing factors of sensitive skin. Endogenous factors are inherent potential host factors that may promote skin sensitivity. ${ }^{3}$ Exogenous factors are external triggering factors for sensitive skin. ${ }^{6}$ (Table 1) shows various endogenous and exogenous factors affecting sensitive skin. However, a combination of quite a few of exogenous and endogenous factors may be responsible for hyperreactivity or sensitivity of the skin. ${ }^{6}$

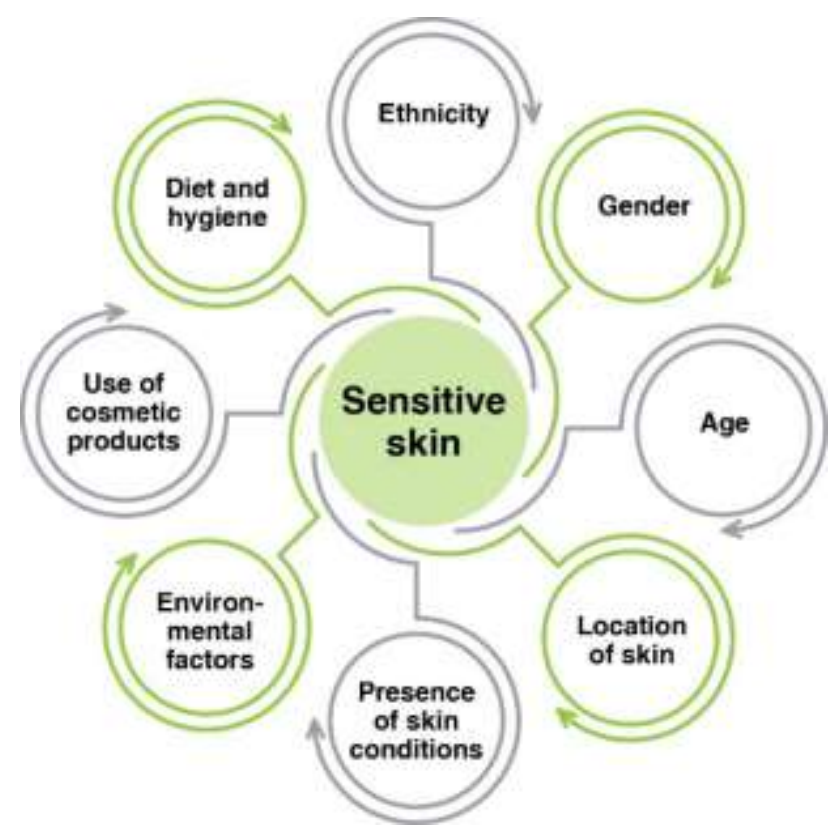

Figure 2: Common predisposing factors of sensitive skin.
Table 1: Triggering factors of sensitive skin.

\begin{tabular}{|c|c|}
\hline Endogenous factors ${ }^{3}$ & Exogenous factors ${ }^{5}$ \\
\hline $\begin{array}{ll}\text { - } & \text { Females } \\
\text { - } & \text { Houng age } \\
\text { - } & \text { Skin pigmentation } \\
\text { - } & \text { Thin stratum corneum } \\
\text { - } & \text { Disrupted skin barrier } \\
\text { - } & \text { Increased sweat glands } \\
\text { - } & \text { Increased epidermal } \\
& \text { innervation } \\
\text { - } & \text { Decreased lipids } \\
\text { - } & \text { High transepidermal } \\
& \text { water loss (TEWL) } \\
\text { - } & \text { Pre-existing skin } \\
& \text { condition (acne, } \\
& \text { rosacea, atopic } \\
& \text { dermatitis) }\end{array}$ & $\begin{array}{l}\text { - Use of cosmetics and } \\
\text { toiletries } \\
\text { - Environmental factors } \\
\text { (light, sun exposure, } \\
\text { heat, pollution) } \\
\text { - Diet (spices, alcohol, } \\
\text { coffee, hot beverages) } \\
\text { - Medications (topical } \\
\text { corticosteroids) } \\
\text { - Cosmetic procedure } \\
\text { (dermabrasion, laser } \\
\text { resurfacing, or facelift) } \\
\text { - Occupational exposure } \\
\text { to chemicals }\end{array}$ \\
\hline
\end{tabular}

\section{CONSENSUS KEY POINT 3}

Apart from the causative agents mentioned above, the panel further added other possible causative factors contributing to sensitive skin, such as excess of cosmetic procedures, continuous exposure to air conditioner, and use of hot and cold water for cleansing; use of tap water at normal temperature is recommended for daily skin care.

\section{Recalling the pathophysiology}

The exact pathophysiology of sensitive skin is poorly understood. However, there exist various hypothetical claims regarding mechanisms and/or factors contributing to the development of sensitive skin. ${ }^{1,2,6}$

\section{Impaired skin barrier}

Stratum corneum, a well-organized multilayered lipid structure consisting of corneocytes, is essential for maintenance of skin barrier function. ${ }^{1,7}$ The integrity of the skin barrier may be breached by physical or chemical trauma, mechanical abrasion, physiological factors such as cutaneous blood flow, stress, sweat gland density, thickness and composition of the stratum corneum, presence of skin conditions such as acne, rosacea, etc.8Impaired barrier function facilitates penetration of irritants or allergens. These substances induce release of inflammatory mediators, which stimulate nerve endings and trigger altered sensations.1Impaired skin barrier contributes to an increase in the permeability of skin, vascular reactivity, and trans epidermal water loss (TEWL). ${ }^{1,7}$ TEWL is considered to be an important indicator of functional state of skin barrier and is generally assessed for the diagnosis of sensitive skin. ${ }^{8}$ 


\section{Neurosensory dysfunction in the skin}

Neurosensory dysfunction in the skin could result from hyperactivity of sensory nerve fibers or exposure of sensory nerve endings due to impaired barrier. Sensory neuroreceptors are responsible for skin sensations such as pain, itching, etc. In sensitive skin, endothelin receptors and pain/cold/heat receptors of the transient receptor potential (TRP) family on $\mathrm{C}$ fibers are believed to act as mediators for altered sensations such as pain, burning, and itch.9Physical factors may interact with these receptors, causing hypersensitivity, thereby contributing to the development of sensitive skin.7A variety of neural changes have been postulated in sensitive skin - altered nerve endings and skin nerve fibre density, increased release of neurotransmitters, chronic nerve ending trauma, slower neurotransmitter removal, etc. ${ }^{7,9}$

\section{Stress}

Skin is sensitive to psychological stress and is considered as an immediate stress perceiver. Clinical evidence shows that stressors affect cutaneous innate and adaptive immune system of the skin.10Stress responses to the skin are mediated mainly by a local hypothalamic-pituitaryadrenal (HPA) axis, peripheral nerve endings, and local skin cells including keratinocytes, mast cells, and immune cells. The factors that are involved in mediating stress responses are corticotropin release hormone, substance P, adrenocorticotropin hormone, cortisol, neurotrophins, catecholamines, etc. Also, mast cells activated by stress producestriggering factors, leading to a vicious cycle of stress-induced inflammatory events. ${ }^{11}$

The panel experts briefly discussed the above-mentioned pathophysiological mechanisms and agreed to their involvement in the development of sensitive skin.

\section{Clinical features of sensitive skin}

Sensitive skin is primarily a subjective perception of skin discomfort characterized by common complaints like burning, stinging, tingling, itching, dryness, and redness. However, there are clearly no visible signs of irritation, which can be attributed to inflammatory or allergic reactions. These signs are believed to be triggered within minutes or hours after using a cosmetic product, physical and/or environmental stimuli. ${ }^{1,3}$ People with sensitive skin vary not only in terms of the signs and symptoms experienced, but also in the degree of sensitivity at different anatomical sites. ${ }^{12}$

Sensitive skin is not restricted to the face but is also frequently reported on non-facial areas of the body such as hands, scalp, feet, neck, torso, and back. Triggering factors may or may not be identical for the face and other major areas of the body. Higher prevalence of sensitive skin on hands suggests impact of environmental factors such sun exposure and frequent use of soaps and shampoos. ${ }^{13}$ A subjective evaluation of sensitivity of skin on different areas of the body was conducted with 400 women; (Table 2) shows the proportion of patients reporting sensitive skin over the various areas of the body. ${ }^{13}$

Table 2: Areas of sensitive skin reported by patients.

\begin{tabular}{|ll|}
\hline Area & Proportion of patients $(\%)$ \\
\hline Face & 85.4 \\
\hline Hands & 57.7 \\
\hline Scalp & 36.2 \\
\hline Feet & 34.2 \\
\hline Neck & 26.8 \\
\hline Torso & 23.0 \\
\hline Back & 20.9 \\
\hline
\end{tabular}

The data has been adapted from Saint-Martory et al ${ }^{13}$

\section{CONSENSUS KEY POINT 4}

The experts further emphasized that the common sites for sensitivity on face are perioral and periorbital regions. Excessive use of toiletries and frequent washing leads to sensitivity of the hands. Sensitive skin is common in all types of skin, but dry skin can aggravate the chances of skin sensitivity. Genitals are also a common site to develop skin sensitivity.

\section{Diagnosis of sensitive skin}

The symptoms of sensitive skin are majorly subjective, and it may or may not manifest as visible signs. Thus, self-assessment questionnaires are valid diagnostic tools in the diagnosis of sensitive skin. ${ }^{1}$ Physical tests are also resorted to, for ruling out any causes of mild or subclinical manifestations of contact allergy. ${ }^{3}$ Objective diagnostic methods for sensitive skin available currently are sensory reactivity tests, irritant reactivity tests, and structural sensitivity tests/dermal function tests. ${ }^{3}$

Sensory reactivity tests provide measure of sensory perception of pain in the absence of visible irritation e.g. sting test with lactic acid. ${ }^{3}$

Irritant reactivity tests provide measure of visible sequelae to irritant exposure (typically dryness or erythema) e.g. cutaneous irritation with detergent. ${ }^{3}$

Structural sensitivity tests assess structural or physiological changes associated with the neurosensory responses in sensitive skin e.g. TEWL measures skin surface water loss and evaluates barrier integrity. ${ }^{3}$

The primary investigation in diagnosing sensitive skin should involve detailed history taking to identify any triggering factors or cosmetic intolerance as the cause of sensitive skin. Also, family history, occupational history, use of specific products, etc. are necessary investigation parameters. Moreover, complete physical examination is an essential part of the investigation to exclude signs of 
inflammation, presence of skin conditions such as contact and atopic dermatitis. 1 Patch testing may be employed to standard allergens and patients' cosmetics and skin care products. $^{6}$

\section{CONSENSUS KEY POINT 5}

The panel indicated that although there are several tests available in real world clinical practice, these tests are not commonly used; the diagnosis is mainly clinical. Some doctors use product challenge test, wherein a sample pack of the cosmeceutical is given to the patient initially and then the products are upgraded gradually. Further, the panel also emphasized on the need of a simple questionnaire, which could aid in diagnosing sensitive skin.

\section{Concept of cosmeceuticals}

The Food, Drug, and Cosmetic Act defines cosmetics by their intended use, as 'articles intended to be rubbed, poured, sprinkled, or sprayed on, introduced into, or otherwise applied to the human body for cleansing, beautifying, promoting attractiveness, or altering the appearance'. ${ }^{14,15}$ Like cosmetics, cosmeceuticals are meant for topical application, but unlike cosmetics they contain potent ingredients. ${ }^{16}$ These ingredients influence the biological function of the skin, nourish the skin, provide medicinal as well as cosmetic benefits to the skin, thereby promoting healthy skin. ${ }^{14,16}$

Commonly used cosmeceutical products, based on function, are cleanser, moisturizer, sunscreen, antioxidants, anti-inflammatory, pigment lightening, collagen repair, exfoliation, hydration/barrier repair products. ${ }^{17}$ Some cosmeceuticals are also claimed to provide other beneficial effects such as more even skin tone, improved skin texture, increased skin radiance, decreased appearance of skin wrinkling, and enhanced anti-aging skin. ${ }^{18}$ Desirable features of cosmeceutical agents are their efficacy, safety, formulation stability, novelty, and metabolism within skin. ${ }^{14}$

While choosing a cosmeceutical, it is important to identify a patient's problems and resolve them with appropriate cosmeceutical product. A patient may have multiple needs, thus a cosmeceutical product with multifactorial benefit would be suitable. ${ }^{17}$ In addition, for a cosmeceutical ingredient, it is essential to evaluate its basic scientific data regarding its pharmacology and biochemistry, in order to validate that the claims made for the cosmeceutical ingredient is supported by body of evidence. ${ }^{19}$

\section{Role of cosmeceutical products in sensitive skin}

A wide range of cosmeceutical products, including cleansers, moisturizers, sunscreens, shampoos, etc. are used for sensitive skin. ${ }^{14}$ These cosmeceuticals have a potential to enhance standard dermatological treatments prescribed for sensitive skin. However, for a suitable cosmeceutical product, it is advised to consult dermatologists, because it is important to complement patient's skin type and manage skin-related issues with appropriate products. ${ }^{16,17}$ Moreover, products with ingredients possessing multifactorial benefits may be applicable for patients with skin requiring multiple needs. ${ }^{17}$ A cosmeceutical does not temporarily beautify the skin. Instead, the presence of biologically active ingredients in it influences biological function of skin, thereby promoting healthy skin. ${ }^{20,21}$ Management of sensitive skin is challenging; therapy for sensitive skin must address various physiological issues involved in the development of skin by improving barrier function, immune hyperactivity, and exaggerated sensory hyperresponsiveness. $^{22}$

\section{CONSENSUS KEY POINT 6}

The panel emphasized on the importance of individualized approach for selection of cosmeceuticals. It is not only the physician's choice; but a patient feedback, which also plays an equally important role in selecting a right cosmeceutical. In addition, the experts highlighted the fact that evidence-based scientific backing for cosmeceutical ingredients is essential.

In the experts' opinion, basic skin care and judicious recommendations of products and cosmetics or cosmeceuticals are effective and is the preferred treatment approach. A wide range of high-quality skin care products or cosmeceuticals are available exclusively for sensitive skin. These products claim to be hypoallergenic, gentle and safe for sensitive skin. However, a methodical step-by-step approach is required for evaluating sensitive skin before recommending any skin care regimen with cosmeceuticals.

\section{Daily skin care cosmeceutical products for sensitive skin}

Skin care products for daily use containing cosmeceutical ingredients have shown to be effective in the management of sensitive skin. ${ }^{22}$ The panel experts discussed a few cosmeceutical products for daily use and described their role in the management of sensitive skin.

\section{Cleansers}

Skin cleansers are surface-active substances that lower the surface tension on the skin and are capable of emulsifying and removing water-insoluble impurities (dirt, sebum, oil from cosmetic products, microorganisms, and exfoliated corneum cells). Cleansers could be emulsifiers, detergents, surfactants or soaps. ${ }^{23}$ Along with the cleansing properties, an ideal cleanser should not cause irritation and should not damage or disrupt the skin. Moreover, it should moisturize the skin, keep the skin surface smooth and preserve homeostatic properties of the skin. ${ }^{23,24}$ 
In people with sensitive and compromised skin, gentle cleansing with liquid facial moisturizing cleansers (e.g. micellar or non-foaming cleansers) which have high rinsibility property is recommended. They should be soap-free, toner-free, and containing nonionic/silicone surfactants. Nonionic surfactants have low irritation potential and $\mathrm{pH}$ compatibility. Silicone surfactants act as both surfactants and emollients. They also possess low irritation potential, and non-comedogenic and hypoallergenic properties. ${ }^{24}$

\section{CONSENSUS KEY POINT 7}

The expert panel suggested that in individuals with sensitive and compromised skin, gentle cleansers should be recommended. Cleansers should be free of abrasives or alcohol, paraben and other preservatives. Besides, experts advised that thermal spring water (TSW) based cleansers could also be preferred for sensitive skin. The experts added that such mild cleansers restore skin barrier function and do not compromise skin barrier integrity and alleviate skin dryness. They also exhibit synergy with prescribed topical or systemic therapy in people with sensitive skin.

In addition, the panel also mentioned that alpha hydroxy acids such as glycolic acid present in cleansers are a peeling agent and should be avoided for sensitive skin. Generally, cleansing two times a day (morning and evening) is advised; and excessive cleansing may cause irritation in patients with sensitive skin.

\section{Moisturizers}

A moisturizer is expected to make skin smooth and soft, repair the skin barrier, increase skin hydration by reducing and preventing TEWL, and restore lipid barrier to hold and redistribute water. ${ }^{25-27}$ An ideal moisturizer should get absorbed immediately into the skin, improve skin appearance (radiance and luminosity), and should be cosmetically elegant, acceptable, and long-lasting. ${ }^{26,27}$ Besides skin hydration, a moisturizer should also have anti-inflammatory, antipruritic, antimitotic, and photoprotective action. ${ }^{27,28}$ Moisturizers are available in the form of creams and lotions, ointments, gels, serums, suspensions, etc. ${ }^{26}$

For sensitive skin, moisturizers containing natural oils (high linoleic acid and low oleic acid ratios) and natural or synthetic ceramides are widely used for sensitive skin (atopic dermatitis). ${ }^{28}$ Also, anti-inflammatory agents have added emollients or humectants, which provide additional barrier repair and control drying of skin. Lipid-rich moisturizers are particularly recommended to be routinely used in all contact dermatitis patients. ${ }^{28}$ Sensitive skin patients are recommended to use moisturizers frequently and overnight application might also be recommended. ${ }^{28}$

\section{CONSENSUS KEY POINT 8}

Expert panel suggested that for sensitive skin, a moisturizer is expected to be hypoallergenic, nonsensitizing, fragrance-free, and non-comedogenic. They highlighted that moisturizers are chosen area wise; creams and lotions are recommended for face and body, respectively. The experts indicated TSW based moisturizers are not only effective for sensitive skin, but also restore skin barrier function and preserve microflora biodiversity. Further, the panel added that moisturizers which are alcohol-based and contain menthol agents and other irritants should be avoided.

\section{Sunscreens}

Sunscreens are effective photo-protective agents. An ideal sunscreen is supposed to have highly efficient filters against both UVB and UVA radiation with SPF of 30 or greater. It should be safe, chemically inert, non-irritating, non-toxic, photostable, and cosmetically elegant.29,30 Ingredients in a sunscreen are broadly classified on the basis of their mode of UV protection - chemical absorbers or physical blockers. ${ }^{31}$ Chemical sunscreens absorb high-energy UV. They contain organic filters such as paraaminobenzoic acid (PABA) derivatives, benzophenones, salicylates, cinnamates, and others like trazone, octocrylene, etc. ${ }^{29,30}$ Physical blockers reflect or scatter light, and contain inorganic filters, specifically titanium dioxide and zinc oxide. ${ }^{29,30}$ Sunscreens are usually incorporated with different types of UV filters, which are photo stable. ${ }^{29}$ Nowadays most sunscreens combine chemical absorbers and physical inorganic blockers to provide broad spectrum UV protection. Using aluminium oxide, zirconium or silicon to coat the particles of blocking agent makes it less reactive. ${ }^{31}$

People who have sensitive skin and/or are intolerant to chemical sunscreens, are usually recommended physical sunscreens containing metal oxides. They are less oily and are suitable for oily and acne-prone skin. ${ }^{32}$ They show better potential for long-term use with high safety profile than chemical sunscreens. Newer broad-spectrum chemical agents, such as bis-ethylhexyloxyphenol methoxyphenyl triazine (BEMT), methylene bisbenzotriazolyl tetramethylbutylphenol (MBBT), and butyl methoxy-dibenzoylmethane (BMDBM) have broadspectrum activity (effective against UVA and UVB rays) and are photostable. These agents claim to have the advantages of instant action, longer duration of protection, antiaging effects, improved cosmetic appearance of the skin (less wrinkles), minimized erythema, and are suitable for children and people with sensitive skin. ${ }^{30}$

Sunscreens are formulated using active and inactive ingredients. Many of these chemicals are associated with allergic reactions and endocrine disrupting activity, especially which easily penetrate the skin. Paraben is one of the inactive ingredients commonly used as 
preservatives in cosmetic products. It shows endocrine disrupting activity and may have negative effects on the reproductive system. ${ }^{33}$ Studies have also evaluated adverse effects of endocrine disrupters on early child development with widespread use of personal care products, including parabens and phthalates. ${ }^{34}$

\section{CONSENSUS KEY POINT 9}

In case of sensitive skin with impaired barrier choosing right sunscreen is imperative. Panel suggested to apply moisturizer before sunscreen application. Further The experts agreed that physical sunscreens are more preferable for sensitive skin. However, physical sunscreen might live a white tinge on the skin, thus some of the panel members prefer using chemical sunscreen or a combination sunscreen with minimal filters thus reducing the chances of exposure to multiple ingredients which could in fact act as a sensitizer.

Further on tinted sunscreen, they are a viable option against protection from visible light. These sunscreens usually come with filters like iron oxides, titanium dioxide and zinc oxide.

On use of sunscreen indoors, Constant exposure to heat and light while cooking can aggravate chances of sensitive skin and pigmentation hence a sunscreen is recommended indoors in such case also. In patients with rosacea and sensitive skin, zinc oxide based, ferric oxide, tinted sunscreen is preferred.

According to the experts, gel-based formulation or matte finish sunscreen is also a preferred choice for sensitive skin. Further, they emphasized that sunscreen should be applied 15 minutes before going outdoors on a regular basis. In children, physical sunscreen is good and safe

Panelists emphasized that apart from sunscreen patients should also be properly educated to utilize photoprotective clothing, seek shade when outdoors, hat and sunglasses to sufficiently protect from both UV radiation and visible light.

\section{Endocrine-disrupting chemicals}

Personal care products contain endocrine-disrupting chemicals (EDCs) which interact directly or indirectly with hormone receptors, thus disrupting the production and action of hormones. ${ }^{35}$ EDCs interfere with normal homeostasis of the body i.e. hormone biosynthesis, their metabolism, and mechanism of action. ${ }^{36}$ These chemicals disturb the endocrine system and are identified to be carcinogenic, immunotoxic, and hepatotoxic to human skin. They are also associated with inflammatory and allergic skin diseases, skin pigmentation, skin cancer, and skin aging. ${ }^{35}$ Exposure to even small concentration of EDCs can cause imbalance of sex hormones and induce excess androgenic stimulation. In addition, elevated androgen levels and excess sebum production have also been implicated in the development of acne by altering follicular growth, colonization of Propionibacterium acnes and inflammation. ${ }^{37}$

\section{CONSENSUS KEY POINT 10}

Panel suggested increased use of EDC in the cosmeceuticals could be one of the reasons why we get more patients with sensitive skin these days.

\section{Shampoos}

In basic formulations of a shampoo, synthetic detergents and conditioners are the only ingredients required for cleansing and manageability of hair. Other ingredients such as foaming agents, thickeners or opacifiers, sequestering agents, $\mathrm{pH}$ adjusters, preservatives and other additives contribute to the stability, presentability, and marketability of the product. ${ }^{38}$ Besides cleaning the scalp of dirt, sebum, sweat, desquamated corneocytes, and greasy residues from oils, lotions or sprays, a shampoo is also supposed to condition hair and soothe scalp irritation in conditions like seborrheic dermatitis. ${ }^{38}$ Medicated shampoos, in addition to regular cleanser, contain tar derivatives, corticosteroids, salicylic acid, sulfur, selenium sulfide, polyvinylpyrrolidone-iodine complex, chlorinated phenols or zinc pyrithione. ${ }^{38}$

Sensitive scalps are more frequently associated with greasy or dry scalps, atopic cause, psychogenic factors, and changes in weather, particularly winter. Inappropriate use of shampoos, frequent shampooing and hair oil application habits are major predisposing factors for scalp sensitivity. ${ }^{39}$

\section{CONSENSUS KEY POINT 11}

The experts emphasized that sensitive scalp condition has a tendency of barrier impairment and increased permeability of the skin. Also, skin hyper-reactivity and non-specific inflammation could be triggered by watersoluble irritants that penetrate the skin. Thus, an expert panel suggested that for sensitive scalp, pH-balanced shampoos containing a mild surfactant are recommended which protects the natural skin barrier. The shampoo ingredients should be anti-inflammatory in action and soothing, i.e. free of potentially irritating additives such as paraben, perfume, and colouring agents. The panel suggested that frequent and regular cleansing with a wellformulated shampoo would not damage hair provided regular conditioner is used.

\section{Role of cosmeceuticals in dermatological disorders}

Cosmeceuticals have been widely used as an adjuvant therapy for various dermatological disorders such as acne, rosacea, atopic dermatitis, and xerotic skin. ${ }^{40-42}$

In patients with acne, synergistic beneficial effects have been demonstrated with the use of cosmeceuticals in 
combination with standard dermatological treatment. ${ }^{41,43}$ In rosacea, appropriate skin care regimen augments therapeutic benefits of medications and helps in repair and maintenance of skin-barrier integrity. Gentle cleansing with foaming face wash and lipid-free cleansers are recommended in patients with rosacea. ${ }^{23,44}$ Moisturizer is recommended to reduce epidermal barrier dysfunction in rosacea. ${ }^{40}$

In atopic dermatitis (AD), use of emollients is usually recommended to restore and preserve the skin barrier. They may play a major role in mild AD and, may further decrease the need for topical glucocorticoids in long-term management of $\mathrm{AD} .{ }^{45} \mathrm{In}$ atopic dry skin, mild cleansing agent with hydration ability is suggested. In xerotic skin, cleansing agents containing humectants and/or emollients, are recommended, that do not cause further dryness. ${ }^{23}$ Thermal spring water, an important ingredient in cosmetics, has low mineral content and a constant physicochemical composition.46 It has been shown to increase cell membrane fluidity, anti-radical properties, stimulatory effects on keratinocyte differentiation, antioxidative, anti-pruritic, immunomodulatory and antiinflammatory effects. ${ }^{46,47}$ Thus, thermal spring water based cosmeceuticals can be a beneficial adjuvant treatment in patients with sensitive skin. ${ }^{46}$

\section{CONSENSUS KEY POINT 12}

The expert panel agreed that add-on therapy with cosmeceuticals is essential for various dermatological disorders. They also added that cosmeceuticals mainly have an adjuvant role in these disorders. They help in improving tolerability to the treatment and facilitate treatment adherence, thereby enhancing treatment compliance. Another important consideration stated by the experts was close monitoring of prescribed cosmeceuticals. In case of any intolerance issues, the patient should be advised to report immediately and discontinue the treatment, which can allow for modifications in the prescription accordingly.

\section{Table 3: Key points 12 from experts' consensus.}

\section{Key points from experts' consensus}

Both subjective and objective perceptions contribute to a complete and clinically-acceptable definition of sensitive skin

Sensitive skin can be observed in all skin types; however dry skin is more susceptible to sensitivity In clinical practice, diagnosis is mainly clinical. A simple questionnaire may prove helpful in diagnosing sensitive skin.

Individualized approach is advised for selection of cosmeceuticals. Patient feedback is important.

Cosmeceutical products for sensitive skin should possess minimum ingredients which have evidencebased scientific backing

Cosmeceuticals as add-on therapy in people with skin disorders are effective and provide treatment benefits

\section{DISCLOSURE}

The expert group discussion was conducted in association with Abbott Healthcare Pvt. Ltd. This article is based on the views expressed during the expert group discussion. The views expressed and discussed in the meetings and stated in this consensus article are the independent views of the authors and not of Abbott Healthcare Pvt. Ltd.

\section{CONCLUSION}

A great magnitude of ambiguity exists in understanding the concept of sensitive skin. Moreover, there are no existing definite and practical frameworks for effective use of cosmeceutical products for management of sensitive skin. This expert consensus paper highlights that appropriate identification of sensitive skin is possible only through both, subjective and objective perception. Selection of a cosmeceutical regimen should be individualized, and a cosmeceutical product containing minimum ingredients should be chosen for management of sensitive skin. Nevertheless, the experts agreed that adjunctive treatment of skin disorders using cosmeceuticals is beneficial.

\section{ACKNOWLEDGEMENTS}

We would like to thank Scientimed Solutions Pvt. Ltd. for assisting in the development of this manuscript. We would like to thank the following physicians who participated in the Consensus meetings:

Dr. Pradeep Kumari, Dr. Rupali Pavaskar, Dr. Ashwini Padmwar, Dr. Kiran Hebbalkar, Dr. Mona Sogani, Dr Aarti Shah, Dr. Kalpana Sarangi, Dr. Punit Saraogi, Dr. Srilata Trasi, Dr Tina Ramchandra, Dr K C Nischal, Dr Urmila Nischal, Dr Aruna Prasad, Dr Kavitha GV, Dr Shubha M Kesari, Dr Sandeep Mahapatara, Dr Jagadish P, Dr Divya Sharma, Dr Rohit Batra, Dr Nivedita Dadu, Dr Gulhima Arora, Dr Indu Balani, Dr Meghna Gupta, Dr Jyoti Sharma, Dr Ritupurna Dash, Dr Chetna Ghura, Dr Mukesh Raj, Dr Vimala Manne, Dr G. Jyotsna, Dr Y. Nikhil Reddy, Dr Mir Mubashir Ali, Dr Smitha Allagadda, Dr K Sai Krishna, Dr Bhargavi. A, Dr V Madhavi Latha.

\section{Funding: No funding sources \\ Conflict of interest: None declared \\ Ethical approval: Not required}

\section{REFERENCES}

1. Duarte I, Silveira JEPS, Hafner MFS, Toyota R, Pedroso DMM. Sensitive skin: Review of an ascending concept. An Bras Dermatol. 2017;92(4):521-5

2. Lev-Tov $H$, Maibach $H$. The sensitive skin syndrome. Indian J Dermatol. 2012;57(6):419-23 
3. Farage MA, Katsarou A, Maibach HI. Sensory, clinical and physiological factors in sensitive skin: a review. Cont Dermat. 2006;55(1):1-14.

4. Kligman AM, Sadiq I, Zhen Y, Crosby M. Experimental studies on the nature of sensitive skin. Skin Res Technol. 2006;12(4):217-22.

5. Brenaut E, Misery L, Taieb C. Sensitive skin in the Indian population: An epidemiological approach. Front Med (Lausanne). 2019;6:29.

6. Pons-Guiraud A. Sensitive skin: A complex and multifactorial syndrome. J Cosmet Dermatol. 2004;3(3):145-8.

7. Inamadar AC, Palit A. Sensitive skin: An overview. Indian J Dermatol Venereol Leprol. 2013;79(1):9-16

8. Warren R, Bauer A, Greif C, Wigger-Alberti W, Jones MB, Roddy MT, et al. Transepidermal water loss dynamics of human vulvar and thigh skin. Skin Pharmacol Physiol. 2005;18(3):139-43.

9. Ständer S, Schneider SW, Weishaupt C, Luger TA, Misery L. Putative neuronal mechanisms of sensitive skin. Exp Dermatol. 2009;18(5):417-23.

10. Hall JM, Cruser D, Podawiltz A, Mummert DI, Jones H, Mummert ME.Psychological stress and the cutaneous immune response: Roles of the HPA axis and the sympathetic nervous system in atopic dermatitis and psoriasis. Dermatol Res Pract. 2012;2012:403908.

11. Chen Y, Lyga J. Brain-skin connection: Stress, inflammation and skin aging. Inflamm Allergy Drug Targets. 2014;13(3):177-90.

12. Farage MA. How do perceptions of sensitive skin differ at different anatomical sites? An epidemiological study. Clin Exp Dermatol. 2009;34(8):e521-30.

13. Saint-Martory C, Roguedas-Contios AM, Sibaud V, Degouy A, Schmitt AM, Misery L. Sensitive skin is not limited to the face. $\mathrm{Br} \mathrm{J}$ Dermatol. 2008;158(1):130-3.

14. Dureja H, Kaushik D, Gupta M, Kumar V, Lather V. Cosmeceuticals: An emerging concept. Indian J Pharmacol. 2005;37:155-9.

15. Center for Safety and Applied Nutrition/Office of Cosmetics and Colors US Food and Drug Administration. Is it a cosmetic, a drug, or both? (Or is it soap?). Plast Surg Nurs. 2008;28(4):195-7.

16. Brandt FS, Cazzaniga A, Hann M. Cosmeceuticals: Current trends and market analysis. SeminCutan Med Surg. 2011;30(3):141-3.

17. Reszko AE, Berson D, Lupo MP. Cosmeceuticals: Practical applications. Dermatol Clin. 2009;27(4):401-16.

18. Draelos ZD. The cosmeceutical realm. Clin Dermatol. 2008;26(6):627-32.

19. Levin J, Momin SB. How much do we really know about our favorite cosmeceutical ingredients? J Clin Aesthet Dermatol. 2010;3(2):22-41.

20. Martin KI, Glaser DA. Cosmeceuticals: The new medicine of beauty. Mo Med. 2011;108(1):60-3.
21. Sarkar R, Arora P, Garg KV. Cosmeceuticals for hyperpigmentation: What is available? J CutanAesthet Surg. 2013;6(1):4-11.

22. Kerscher, Martina \&Buntrock, Heike. 2017. Treatments for sensitive skin. In: Honari G. Andersen RM. Maibach HI (ed.) Sensitive Skin Syndrome, Second Edition.

23. Mukhopadhyay P. Cleansers and their role in various dermatological disorders. Indian J Dermatol. 2011;56(1):2-6.

24. Kuehl BL, Fyfe KS, Shear NH. Cutaneous cleansers. Skin Therapy Lett. 2003;8(3):1-4.

25. Spada F, Barnes TM, Greive KA. Skin hydration is significantly increased by a cream formulated to mimic the skin's own natural moisturizing systems. Clin CosmetInvestig Dermatol. 2018;11:491-7.

26. Draelos ZD. The science behind skin care: Moisturizers. J Cosmet Dermatol. 2018;17(2):13844.

27. Sethi A, Kaur T, Malhotra SK, Gambhir ML. Moisturizers: The slippery road. Ind J Dermatol. 2016;61(3):279-87.

28. Purnamawati S, Indrastuti N, Danarti R, Saefudin T. The role of moisturizers in addressing various kinds of dermatitis: A Review. Clin Med Res. 2017;15(34):75-87.

29. Mancuso JB, Maruthi R, Wang SQ, Lim HW. Sunscreens: An update. Am J Clin Dermatol. 2017;18(5):643-650.

30. Latha MS, Martis J, Shobha V, Sham Shinde R, Bangera S, Krishnankutty B, et al. Sunscreening agents: a review. J Clin Aesthet Dermatol. 2013;6(1):16-26.

31. Moloney FJ, Collins S, Murphy GM. Sunscreens: Safety, efficacy and appropriate use. Am J Clin Dermatol. 2002;3(3):185-91.

32. More BD. Physical sunscreens: on the comeback trail. Indian J Dermatol VenereolLeprol. 2007;73(2):80-5.

33. Maipas S, Nicolopoulou-Stamati P. Sun lotion chemicals as endocrine disruptors. Hormones (Athens). 2015;14(1):32-46.

34. Teitelbaum S, Wolff M, Hauptman M, Galvez M, Brenner B, Ye X, et al. Personal care product use and endocrine disrupting chemicals in urban minority children. Epidemiology. 2009;20(6):S20.

35. $\mathrm{Ju} \mathrm{Q}$, Zouboulis CC. Endocrine-disrupting chemicals and skin manifestations. Rev Endocr Metab Disord. 2016;17(3):449-57.

36. Diamanti-Kandarakis E, Bourguignon JP, Giudice LC, Hauser R, Prins GS, Soto AM, et al. Endocrinedisrupting chemicals: An Endocrine Society scientific statement. Endocr Rev. 2009;30(4):293342.

37. Mazioti M. Chemical environmental factors: Can they affect acne? Indian $\mathbf{J}$ Dermatol Venereol Leprol. 2017;83(5):522-4.

38. D'Souza P, Rathi SK. Shampoo and conditioners: What a dermatologist should know? Indian J Dermatol. 2015;60(3):248-54. 
39. Godse K, Zawar V. Sensitive scalp. Int J Trichology. 2012;4(2):102-4.

40. Draelos ZD. Cosmeceuticals for rosacea. Clin Dermatol. 2017;35(2):213-7.

41. Kantikosum K, Chongpison Y, Chottawornsak N, Asawanonda P. The efficacy of glycolic acid, salicylic acid, gluconolactone, and licochalcone A combined with $0.1 \%$ adapalene vs adapalene monotherapy in mild-to-moderate acne vulgaris: A double-blinded within-person comparative study. Clin CosmetInvestig Dermatol. 2019;12:151-61

42. Goh CL, Noppakun N, Micali G, Azizan NZ, Boonchai W, Chan Y, et al. Meeting the challenges of acne treatment in Asian patients: A review of the role of dermocosmetics as adjunctive therapy. J Cutan Aesthet Surg. 2016;9(2):85-92.

43. Araviiskaia E, Lopez Estebaranz JL, Pincelli C. Dermocosmetics: Beneficial adjuncts in the treatment of acne vulgaris. J Dermatolog Treat. 2019:1-8.

44. Del Rosso JQ, Baum EW. Comprehensive medical management of rosacea: An interim study report and literature review. J Clin Aesthet Dermatol. 2008;1(1):20-5.

45. Lee JH, Jung KE, Lee YB, Kim JE, Kim HS, Lee $\mathrm{KH}$ et al. Use of emollients in atopic dermatitis: a questionnaire survey study. Ann Dermatol. 2014;26(4):528-31.

46. Merial-Kieny C, Castex-Rizzi N, Selas B, Mery S, Guerrero D. Avène Thermal Spring Water: an active component with specific properties. J Eur Acad Dermatol Venereol. 2011;25(1):2-5.

47. Aries MF, Hernandez-Pigeon H, Vaissière C, Delga H, Caruana A, Lévêque M, et al. Anti-inflammatory and immunomodulatory effects of Aquaphilusdolomiae extract on in vitro models. Clin Cosmet Investig Dermatol. 2016;9:421-34.

Cite this article as: Kohli M, Pant H, Dixit R, Parthasaradhi A. Expert consensus on defining sensitive skin and role of cosmeceuticals: an Indian perspective. Int J Res Dermatol 2020;6:827-36. 